\title{
Analysis of Electron and Microwave Behavior in Microwave Discharge Neutralizer*
}

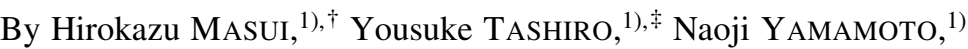 \\ Hideki NAKASHIMA ${ }^{1)}$ and Ikkoh FUNAKI ${ }^{2)}$ \\ ${ }^{1)}$ Department of Advanced Energy Engineering Science, Kyushu University, Fukuoka, Japan \\ ${ }^{2)}$ Japan Aerospace Exploration Agency, Kanagawa, Japan
}

(Received July 27th, 2005)

\begin{abstract}
In the MUCES-C mission conducted by JAXA (Japan Aero Exploration Agency), a microwave neutralizer is mounted with a microwave ion engine on the HAYABUSA space probe. The neutralizer consists of an L-shaped antenna to inject microwaves and samarium cobalt magnets to provide ECR (electron cyclotron resonance). Plasma production of a higher density than the cutoff density is expected in the discharge chamber, but the neutralizer is so small that high-precision measurements using a probe are difficult. To clarify the plasma production mechanism in the microwave neutralizer, numerical analysis was conducted using a code coupling PIC (particle-in-cell) method, and a FDTD (finitedifference-time-domain) method. This paper describes effects caused by varying magnetic field configuration and antenna position in the neutralizer. The calculation results show that bringing the antenna closer to the ECR region is effective for plasma production.
\end{abstract}

Key Words: Electric Propulsion, Microwave Discharge, Neutralizer, Simulation

\author{
Nomenclature \\ $|B|$ : magnetic field strength \\ $B_{\mathrm{e}}$ : magnetic field strength at electron position \\ $|E|$ : electric field strength of microwave \\ $E_{\mathrm{i}}$ : ionization energy \\ $t$ : time
}

\section{Introduction}

The HAYABUSA space probe developed by the Japan Aerospace Exploration Agency (JAXA) uses, four ion engines employing microwave discharge as the main thrust$\mathrm{er},{ }^{1)}$ to maintain electric neutralization on a satellite using an ion engine system, a neutralizer is generally used to supply an equal number of electrons to the ejected ions. Neutralizers with microwave discharge are used by HAYABUSA. The neutralizer consists of an L-shaped antenna to inject the microwaves, a magnetic circuit to form a magnetic mirror, and Electron Cyclotron Resonance (ECR) to generate plasma. Electrons are produced by the difference in the potential between the ion beam and neutralizer. The performance of the neutralizer, such as the electron current and durability, are satisfactory for practical use, but the plasma production mechanism is not fully understood. In particular, it is very important to understand the energy increment process for ionization collision. While experimen-

(C) 2006 The Japan Society for Aeronautical and Space Sciences

*Presented at Symposium on Space Transportation 2005, January, 19th, 2005, Kanagawa, Japan.

†Presently Department of Electrical Engineering, Kyushu Institute of Technology, Fukuoka, Japan.

${ }^{\ddagger}$ Presently IHI Aerospace, Tokyo, Japan. tal production of plasma with a larger density than the cutoff density is expected, ${ }^{2)}$ the neutralizer is so small that highprecision measurements using probes are difficult. Numerical simulation is an effective tool for understanding phenomena in the neutralizer.

So far, numerical simulation of the microwave ion engine has been conducted by Hirakawa ${ }^{3)}$ but no report has been made on numerical simulation of a microwave neutralizer. This is because plasma production in the microwave neutralizer is complex due to the interplay between ECR, antenna and mirror magnetic field. This interplay is interesting from the viewpoint of plasma engineering in general.

This research examines the effects of changes in the magnetic field configuration and antenna position on the electron energy and ionization collision. For this purpose, we developed a code coupling particle-in-cell (PIC) method and finite difference time domain (FDTD) method. ${ }^{4}$ Adopting these methods has advantages in that the PIC method can treat collision process and the distribution function, while the FDTD method can analyze absorption of microwave in plasma. Analysis of the neutralizer using this code will also be very useful for developing microwave plasma sources using antennas. ${ }^{5,6)}$

This report first explains the details of the PIC and FDTD methods. Then, the calculation results for electron energy are shown when the magnetic configuration and antenna position are changed. The electron energy distribution function (EEDF) and ionization collision position are also discussed.

\section{Calculation Method}

The PIC method is used for analysis of plasma behavior in 
the discharge chamber. The effects of the electromagnetic field on plasma particles are calculated by linear interpolation. The basic equation for the method is the motion equation for electrons as follows:

$$
m \frac{d \boldsymbol{v}}{d t}=e(\boldsymbol{E}+\boldsymbol{v} \times \boldsymbol{B})
$$

where, $m, \boldsymbol{v}$ and $e$ are the mass, velocity and charge of electron, respectively. $\boldsymbol{E}$ and $\boldsymbol{B}$ are the electric field of the microwave and the magnetic flux density formed by magnets. Integration with respect to time is conducted by the leap-frog method. In the PIC method, electron-neutral collisions such as elastic, excitation and ionization processes are included and are treated by the Monte Carlo method and Null-collision method. ${ }^{7)}$ Electron energy losses are different among these collision processes. The present calculation treats xenon as a neutral particle. Cross-section data used in the code are taken from Ref. 8).

Microwave propagation is analyzed using the FDTD method. The basic equation for the method is Maxwell's equation as follows:

$$
\begin{aligned}
& \nabla \times \boldsymbol{E}=-\mu_{0} \frac{\partial \boldsymbol{H}}{\partial t} \\
& \nabla \times \boldsymbol{H}=\varepsilon_{0} \frac{\partial \boldsymbol{E}}{\partial t}+\boldsymbol{J}
\end{aligned}
$$

where, $\mu_{0}$ and $\varepsilon_{0}$ are the permeability and permittivity of a vacuum. $\boldsymbol{H}$ is the magnetic field of the microwave. $\boldsymbol{J}$ is the current density calculated from the behavior of the electron. Electromagnetic field components are defined at specific points of unit cells as suggested by Yee. ${ }^{9)}$ The electric field is defined a half-cell from the magnetic field. This arrangement permits natural satisfaction of rotation with respect to space.

In the coupling code, time evolution is solved by exchanging the electric field and current density between the PIC and FDTD methos. ${ }^{10)}$ Figure 1 shows a flowchart for the code. The calculation coordinate system is the 3D Cartesian coordinate system. An advantage of using the $3 \mathrm{D}$ code is the ability to include $3 \mathrm{D}$ structures, such as the L-shaped antenna.

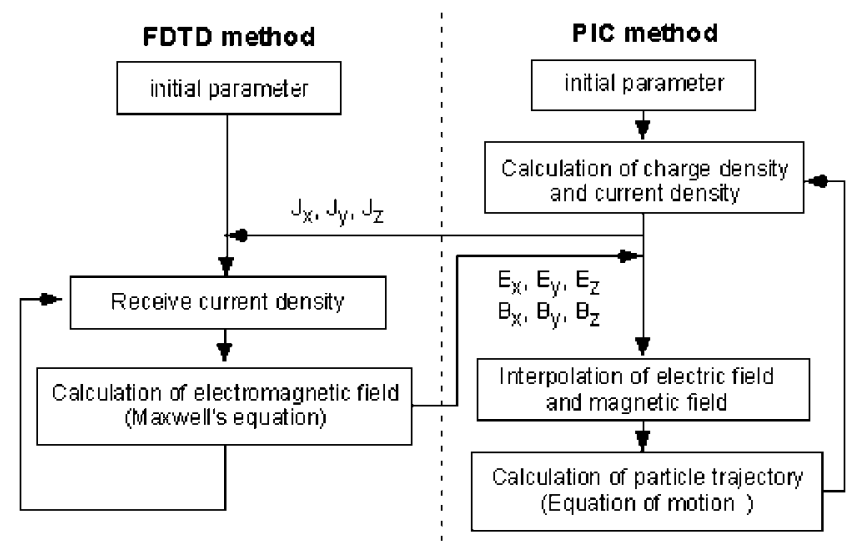

Fig. 1. Flow chart of coupling code.
Since we focus here on interactions between electrons, microwaves and magnetic fields, only electron motion is accounted for in the calculation. The electron gains energy from the microwaves and ionizes neutral particles and then plasma production in the ECR discharge is dominated by electron behavior, so ions are treated as background. The coupling code does not consider the static electric field induced by charge separation between electrons and ions and the Poisson equation is not solved. Consequently, we must simulate the sheath at the boundary of the conductor and magnet. We introduce the sheath model ${ }^{11)}$ as follows. For electrons reaching the boundary, 1) an electron with an energy of less than $20 \mathrm{eV}$ is reflected at the surface boundary, and 2) an electron with an energy larger than $20 \mathrm{eV}$ is removed from the calculation region. The value of $20 \mathrm{eV}$ is based on the plasma potential obtained from the experimental results. ${ }^{12)}$ For the microwave boundary condition, a perfect reflection condition is adopted on a yoke and a perfect conductor. Mur's first absorption boundary condition $^{13)}$ is imposed at the orifice.

\section{Calculation Geometry and Conditions}

Figure 2 shows a schematic for the calculation geometry. For the coordinate system, the $Z$ axis is in the axial direction as shown in Fig. 2. Here, we take the following three cases:

Case 1: Antenna close to ECR region

Case 2: No ECR region in discharge chamber

Case 3: Antenna away from ECR region

Case 1 is based on the HAYABUSA neutralizer. The external magnetic field is formed by six Sm-Co magnets located discretely around the discharge chamber. The microwaves are fed by the L-shaped antenna through a coaxial line formed by the antenna and back yoke. The microwaves mode is TEM in the coaxial line. The antenna length is $8 \mathrm{~mm}$ in the discharge chamber. In Case 2, three magnets are arranged so as not to generate the ECR region. The tip of the antenna in Case 3 is $3 \mathrm{~mm}$ away from that in Case 1.

Figure 3(a) shows the external magnetic field configuration in cases 1 and 3 . The ECR region $(0.15 \mathrm{~T})$ is near the back yoke. The external magnetic field configuration for Case 2 shown in Fig. 3(b) has asymmetry and the magnetic field strength $|B|$ near the antenna is approximately $0.04 \mathrm{~T}$.

The calculation conditions are shown in Table 1. The time steps are different between the PIC and FDTD methods. The reasons are: 1) stability conditions are different between the methods, and 2) the time step in PIC method is increased to decrease the calculation load. Generally speaking, the calculation time to reach the steady-state is about $1-5 \mu \mathrm{s}$. If the time step mentioned above is used, the calculation load to obtain the steady-state condition will be impractically large. The purpose of the present research is analysis of electron behavior in a microwave neutralizer, so the calculation time is set to $200 \mathrm{~ns}(400,000$ steps $)$ to confirm the bounce motion of electrons in the magnetic mirror. The microwave frequency is $4.25 \mathrm{GHz}$ and the input 


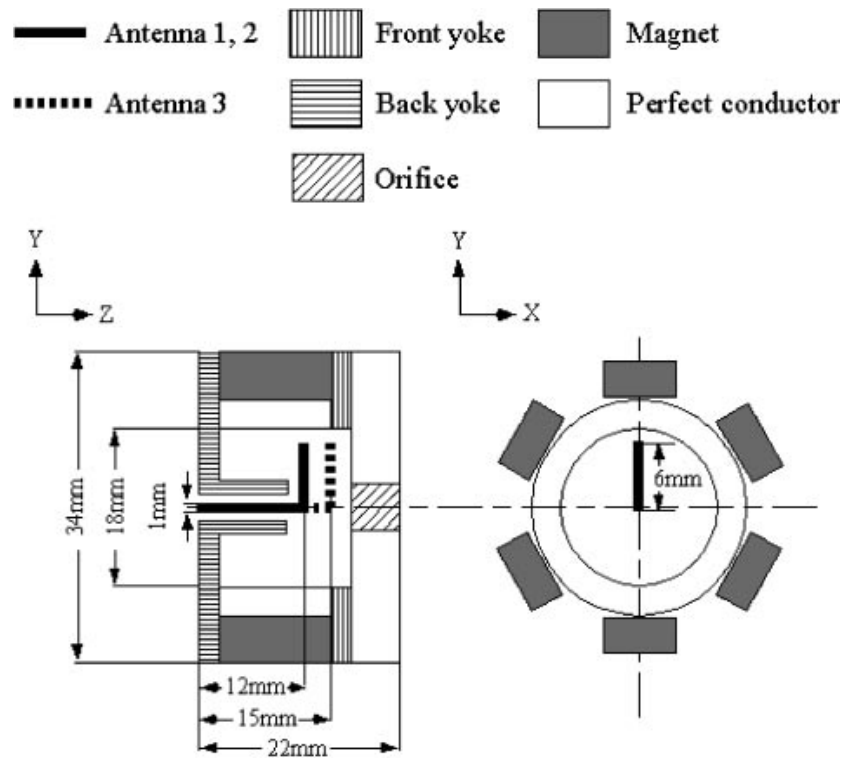

(a) Cases 1 and 3

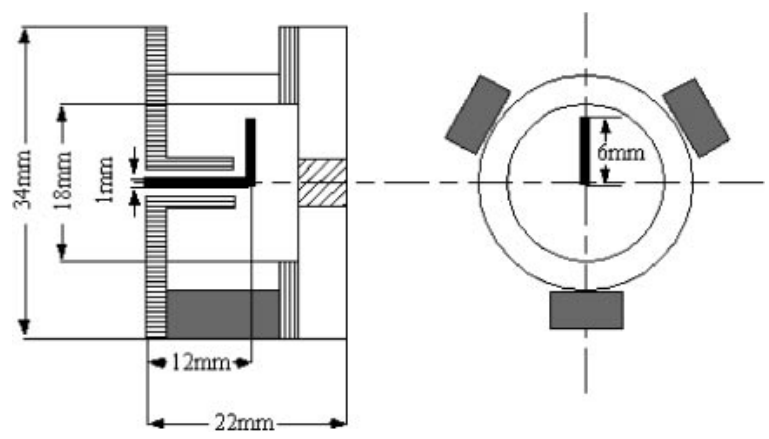

(b) Case 2

Fig. 2. Calculation geometry.

power is $5 \mathrm{~W}$. The neutral particle density is set to $3.21 \times 10^{20} \mathrm{~m}^{-3}$ because the pressure in the discharge chamber is estimated to be about $1.33 \mathrm{~Pa}$. The neutral particle density is assumed to be uniform in the discharge chamber. The initial electron distribution is assumed to be uniform in the discharge chamber and the velocity distribution is Maxwellian with a peak of $1 \mathrm{eV}$. The initial particles are 2,846,000 each representing 125,000 real particles.

\section{Calculation Results}

\subsection{Spatial distribution of electric field strength}

Figure 4 shows the spatial distribution of electric field strength $|E|$. As seen, $|E|$ is high near the antenna for all cases. Cases 1 and 2 have very similar spatial distributions of $|E|$ because the antenna configuration is the same. The plasma effect calculated from the current density is small. In Case 1 , the value of $|E|$ in the ECR region exceeds $9000 \mathrm{~V} / \mathrm{m}$, but it is only $1000-3000 \mathrm{~V} / \mathrm{m}$ for Case 3 .

\subsection{Spatial distribution of electron energy}

The spatial distribution of electron energy $\overline{E_{\mathrm{e}}}$ at $200 \mathrm{~ns}$ is shown in Fig. 5. Here, $\overline{E_{\mathrm{e}}}$ of grid point (m) is given as follows:
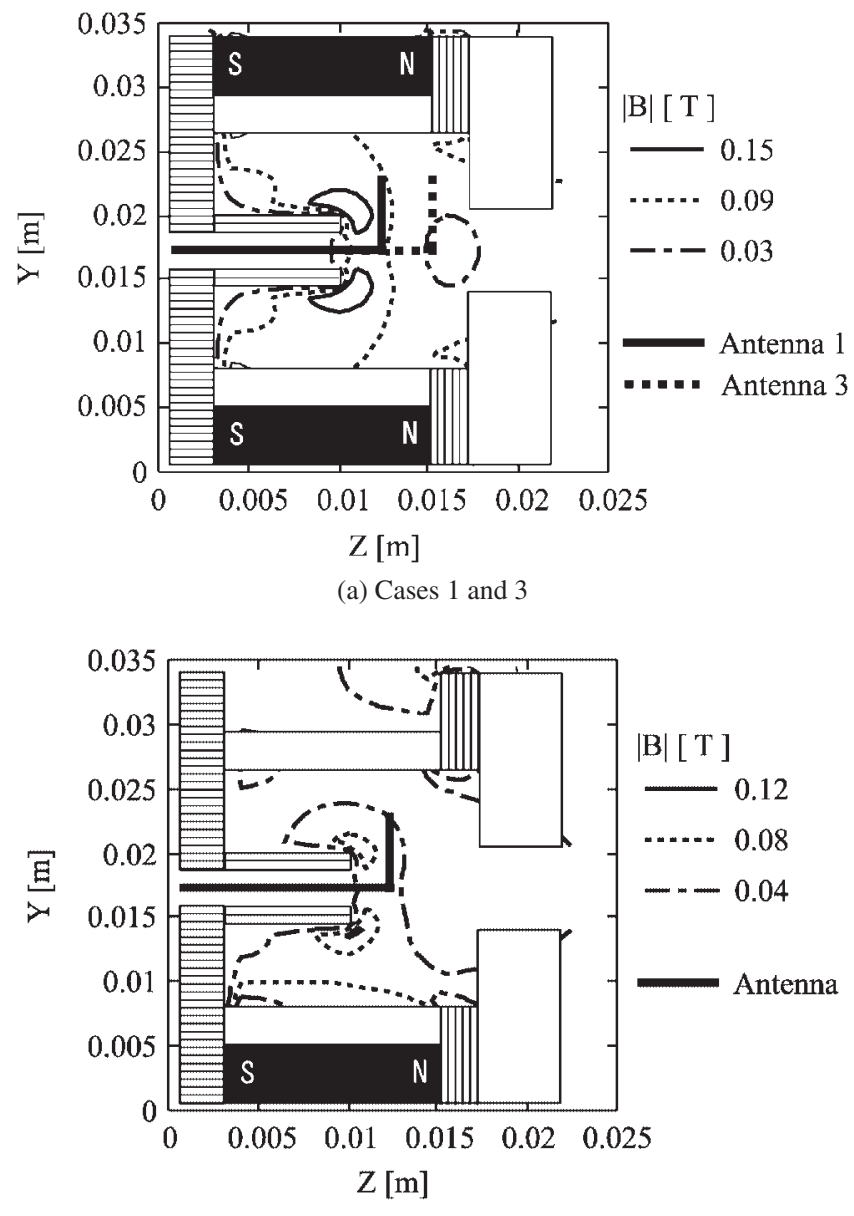

(b) Case 2

Fig. 3. Magnetic field configuration.

Table 1. Calculation conditions.

\begin{tabular}{lc}
\hline Time step (FDTD) & $5.0 \times 10^{-13} \mathrm{~s}$ \\
Time step (PIC) & $1.0 \times 10^{-11} \mathrm{~s}$ \\
\hline Microwave frequency & $4.25 \mathrm{GHz}$ \\
\hline Microwave power & $5 \mathrm{~W}$ \\
\hline Propellant & Xenon \\
\hline Neutral particle density & $3.21 \times 10^{20} \mathrm{~m}^{-3}$ \\
\hline Initial density & $1.0 \times 10^{17} \mathrm{~m}^{-3}$ \\
\hline Initial energy & Maxellian \\
\hline
\end{tabular}

$$
\overline{E_{\mathrm{e}}}(m)=\sum_{i} E_{\mathrm{e} i} W_{i} / \sum_{i} W_{i}
$$

where, $E_{\mathrm{e} i}$ is the electron energy for the $i$-th electron, and $W_{i}$ indicates the weighting factor allotted to the grid point $(\mathrm{m})$ for the $i$-th electron. $W_{i}$ is calculated using the PIC method. The symbol $\Sigma$ means the summation over electrons in the vicinity of the grid point.

For Case 1 shown in Fig. 5(a), $\overline{E_{\mathrm{e}}}$ increases in the ECR region and near the antenna. The value of $\overline{E_{\mathrm{e}}}$ inside the ECR region is higher than $3 \mathrm{eV} . \overline{E_{\mathrm{e}}}$ for Case 2 (Fig. 5(b)) is highest near the antenna, but its value is lower than Case 1. The increase in $\overline{E_{\mathrm{e}}}$ in Case 3 (Fig. 5(c)) is seen in the 


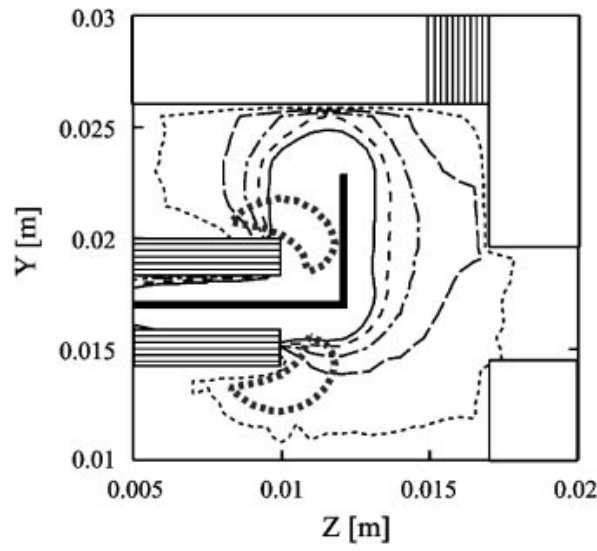

(a) Cases 1 and $3(t=40 \mathrm{~ns})$

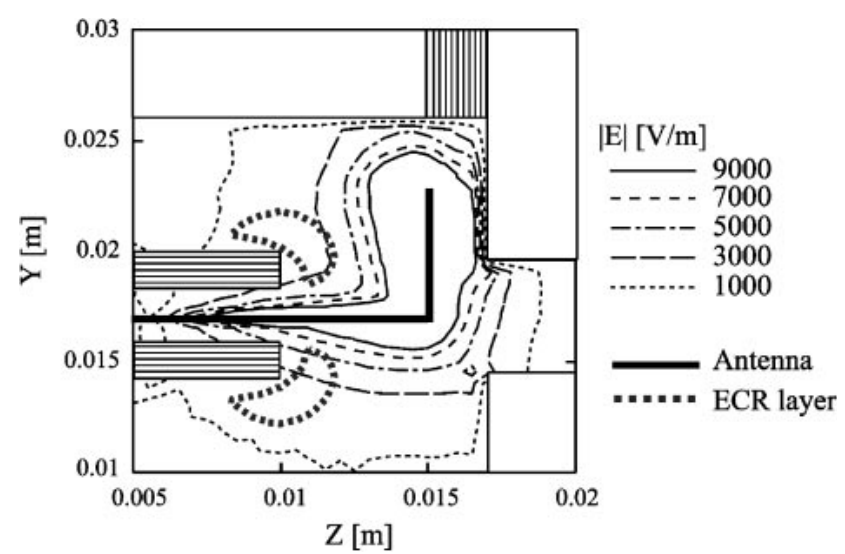

(b) Case $2(t=42.5 \mathrm{~ns})$

Fig. 4. Distribution of electric field strength.

region along the antenna, but not in the ECR region. The result for Case 3 indicates that the microwave electric field strength in the ECR region is low relative to Case 1 (Fig. 4(b)).

\subsection{Electron energy distribution function (EEDF)}

This section compares EEDF among the three cases. EEDF is averaged over $200 \mathrm{~ns}$. In this calculation, EEDF is calculated in three regions (I-III) of a $2.5-\mathrm{mm}$ cube as shown in Fig. 6. Region I includes the ECR region, while the antenna is included in Region II for Cases 1 and 2 and in Region III for Case 3. The ionization energy $E_{i}$ $(=12.31 \mathrm{eV})$ of xenon is shown in Fig. 7. From the EEDF of Case 1 shown in Fig. 7(a), the number of electrons with an energy higher than $E_{i}$ is large in Region II. The EEDF of Case 2 (Fig. 7(b)) shows that high-energy electrons exist in Region II. Figure 7(c) shows the EEDF for Case 3. The number of electrons with an energy higher energy than $E_{i}$ is large in Region III (the antenna), but the high-energy electrons are scarce in Region I (ECR region).

Figure 8 shows an electron trajectory for Case 1. First, the electron moves along a line of magnetic force from the initial position $(X=0.017 \mathrm{~m}, Y=0.019 \mathrm{~m}, Z=0.013 \mathrm{~m})$ near the antenna, then it is reflected back from the front yoke, and moves towards the back yoke. The electron crosses the ECR region and is then reflected back from the back yoke. As a result, the electron bounces twice between the

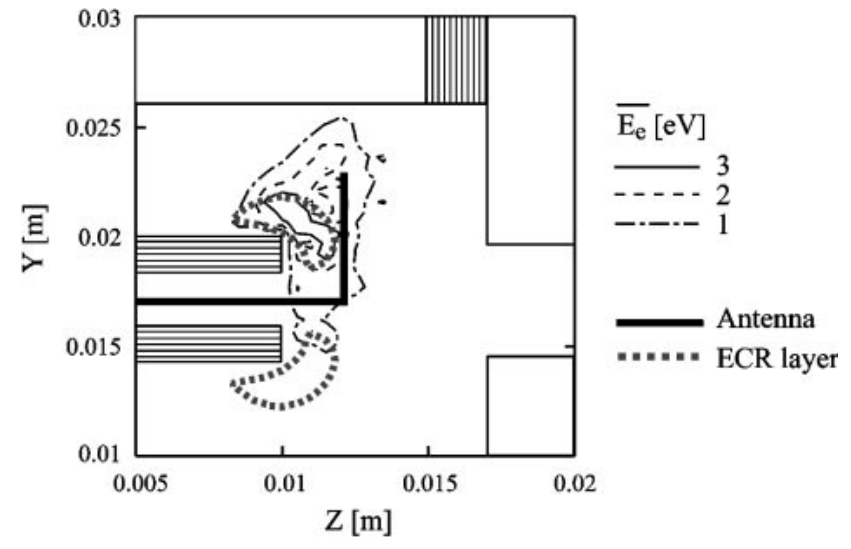

(a) Case 1

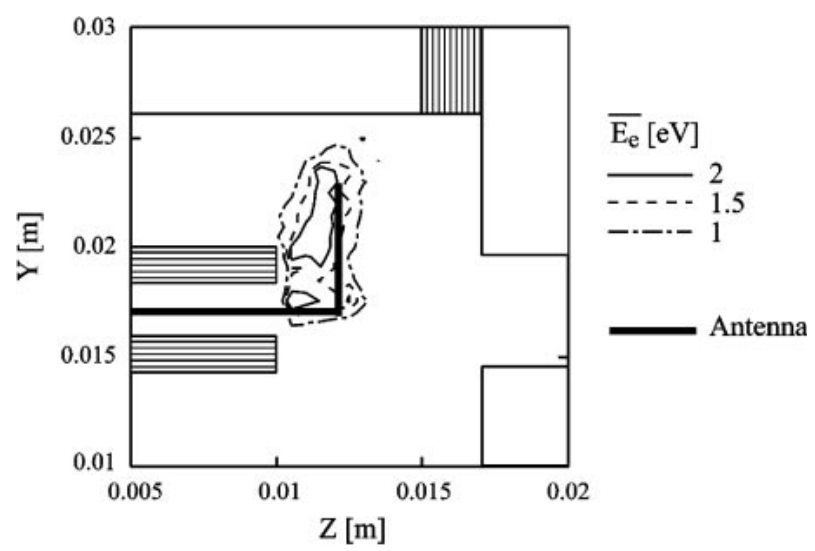

(b) Case 2

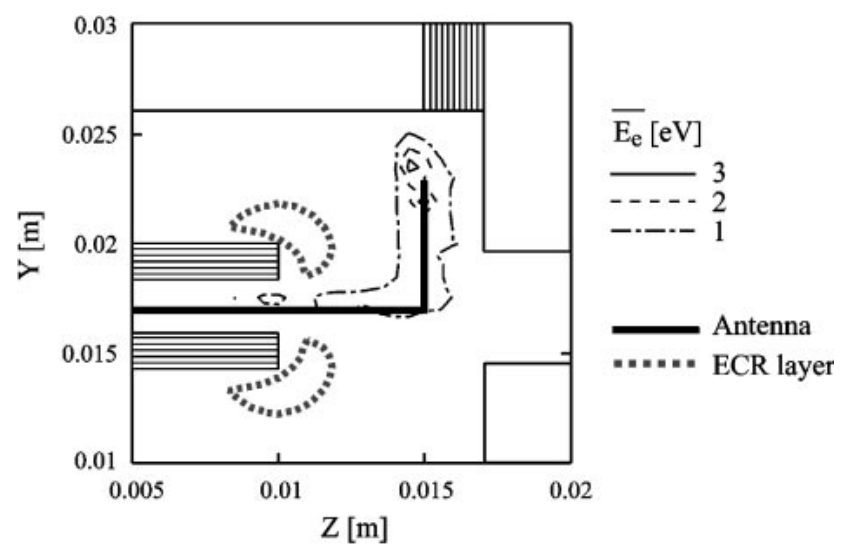

(c) Case 3

Fig. 5. Distribution of electron energy $\overline{E_{\mathrm{e}}}(t=200 \mathrm{~ns})$.

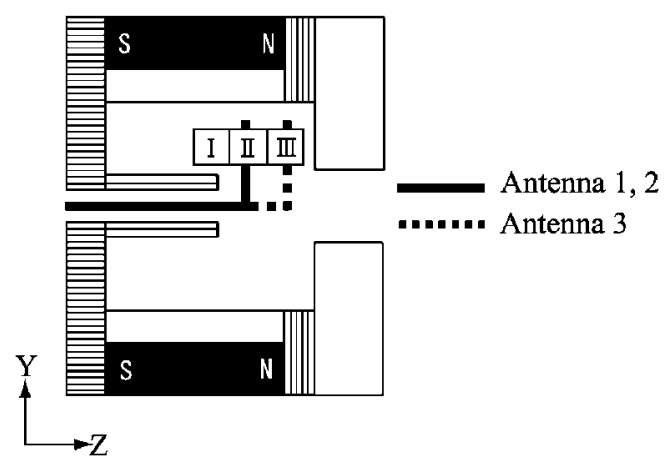

Fig. 6. Definition of calculation region for EEDF. 


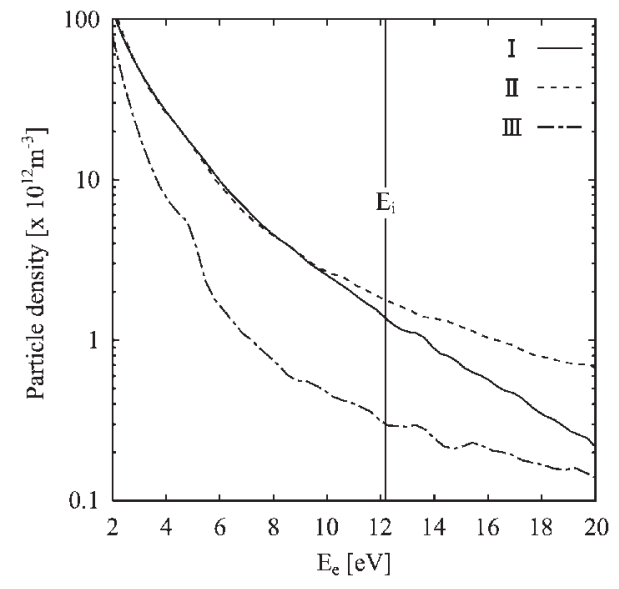

(a) Case 1

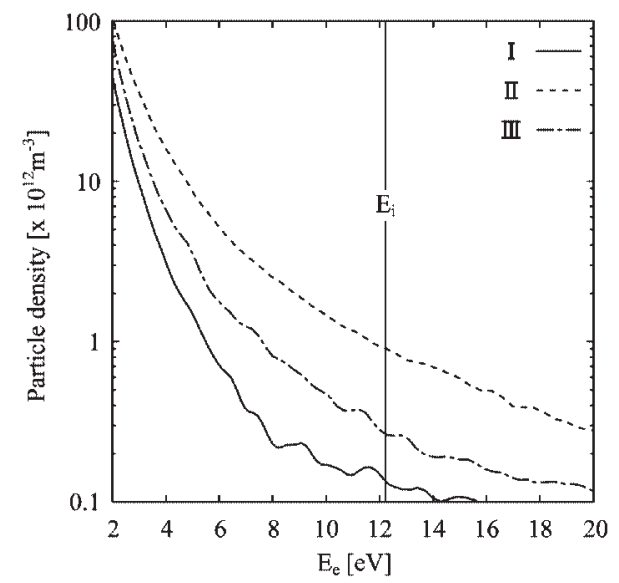

(b) Case 2

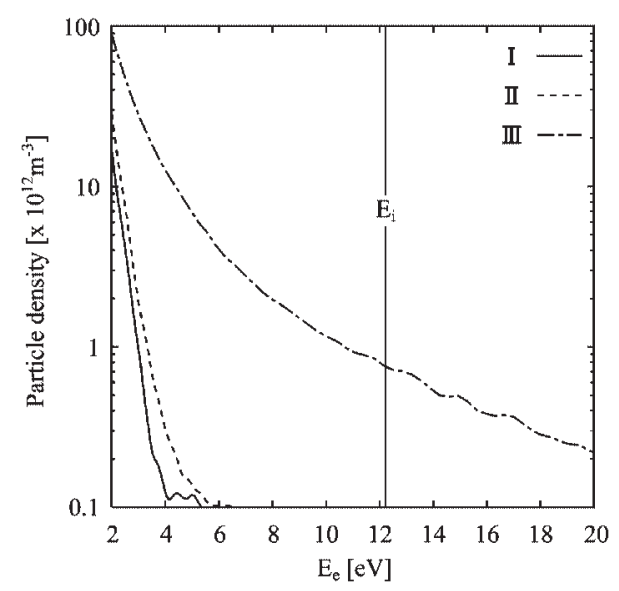

(c) Case 3

Fig. 7. EEDF averaged over $200 \mathrm{~ns}$.

yokes for $100 \mathrm{~ns}$. Figure 9 shows the time variations of the electron energy $E_{\mathrm{e}}$ and magnetic field strength $B_{\mathrm{e}}$ that the electron experiences during the bounce. From the relationship between $B_{\mathrm{e}}$ and $E_{\mathrm{e}}$ for electron behavior, we can see that the electron energy $E_{\mathrm{e}}$ increases rapidly to approximately $30 \mathrm{eV}$ when the electron crosses the ECR region at $30 \mathrm{~ns}$. Immediately after that, $E_{\mathrm{e}}$ decreases due to ionization collision. However, $E_{\mathrm{e}}$ increases again to $10 \mathrm{eV}$ by passing near the antenna at $65 \mathrm{~ns}$.

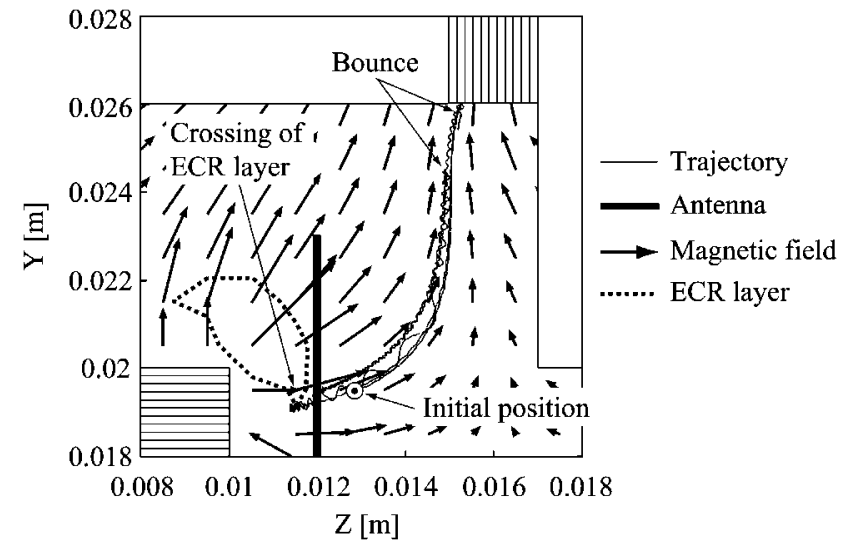

Fig. 8. Trajectory of electrons and lines of magnetic force at $X=$ $0.017 \mathrm{~m}$.

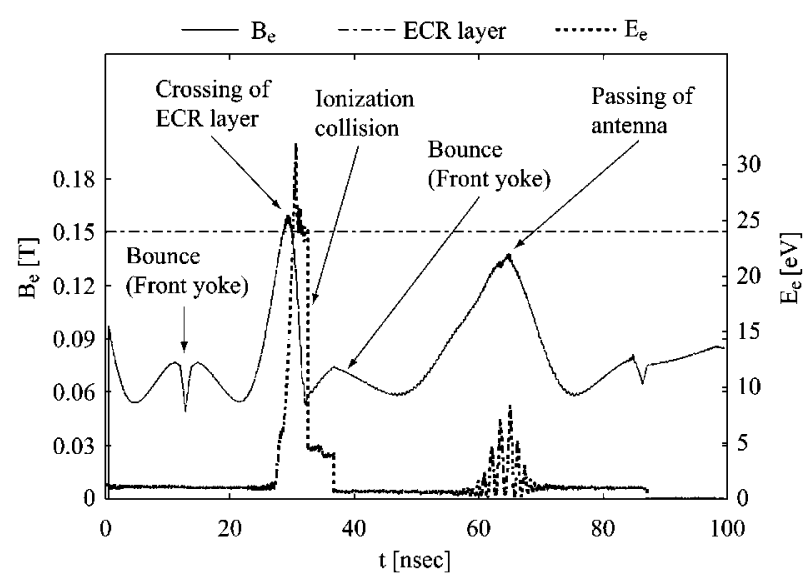

Fig. 9. Time variations of electron energy $E_{\mathrm{e}}$ and magnetic field strength $B_{\mathrm{e}}$.

\subsection{Ionization collision}

All ionization collision positions occurring within $200 \mathrm{~ns}$ are shown in Fig. 10. Ionization collisions in Case 1 in Fig. 10(a) are seen both in the ECR region and antenna, as well as beyond the antenna tip. Ionization collisions in Case 2 (Fig. 10(b)) occur at the antenna tip, but the number of collision is less than in Case 1. Figure 10(c) shows the result for Case 3. Ionization collisions occur in the region along the antenna, but are not seen in the ECR region unlike Case 1. In Cases 1 and 3, the number of ionization collision decreases in the area beyond $Z=0.015 \mathrm{~m}$, because electron motion along $Z$ is prevented because the lines of magnetic force are perpendicular to the $Z$ axis at $Z=0.015 \mathrm{~m}$ as shown in Fig. 8.

\section{Discussion}

As shown in Fig. 10(a), ionization collisions can be seen beyond the antenna as well as in the ECR region and near the antenna. From the electron trajectory (Fig. 8), electrons between the front and back yokes are confined by the mirror magnetic field and gain energy from the microwaves. These high-energy electrons move along the lines of magnetic 

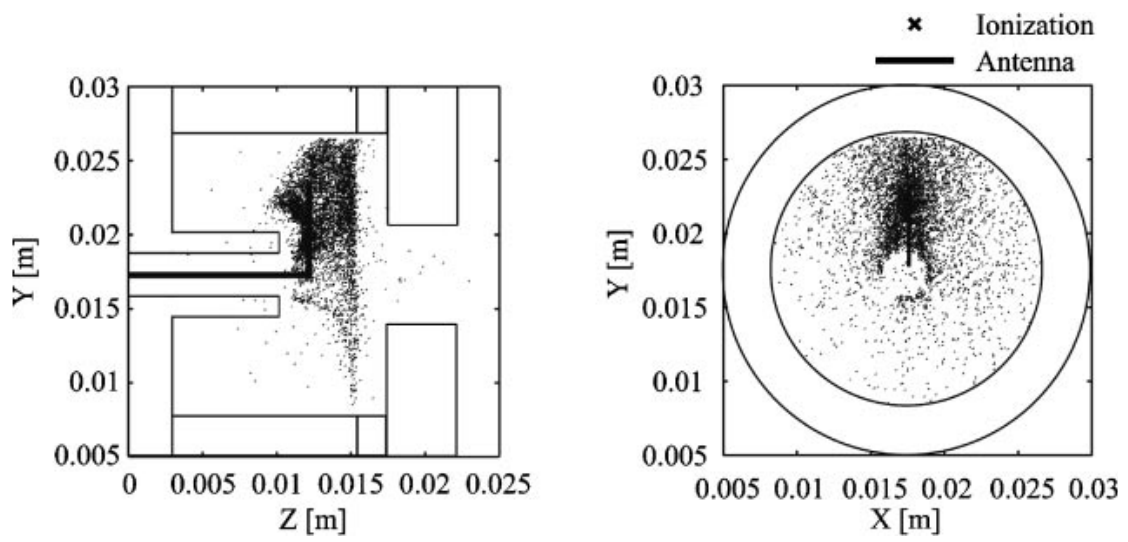

(a) Case 1
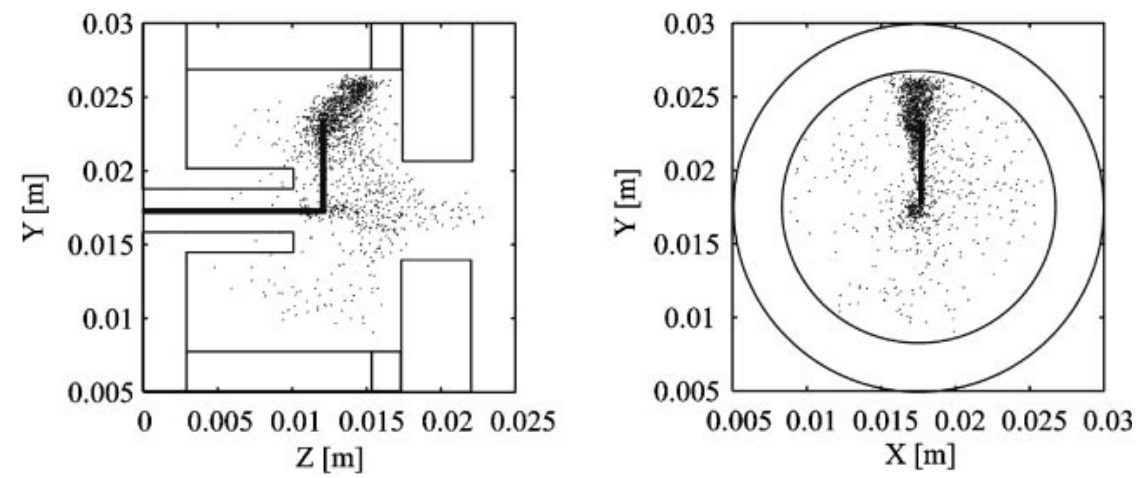

(b) Case 2
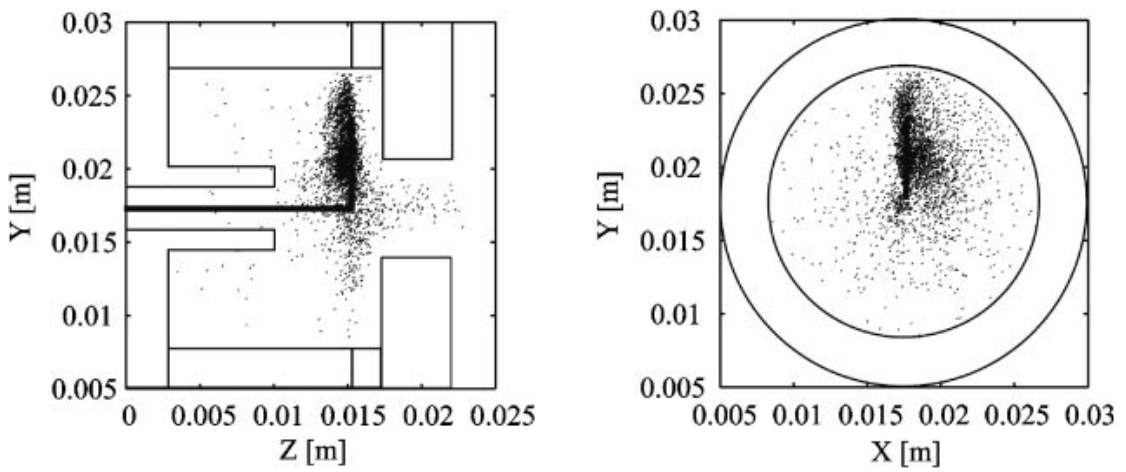

(c) Case 3

Fig. 10. Position of ionization collision $(t=200 \mathrm{~ns})$.

force and ionize neutral particles. This is why ionization collision sites extend beyond the antenna. Accordingly, plasma production by the microwave neutralizer seems to be maintained by the cycle in which electrons confined in the mirror magnetic field gain energy from the microwave, then lose the energy by ionization collision, and then gain energy again from the microwaves.

Figure 10(b) (Case 2) shows that ionization collisions can occur by electrons directly energized from the electric field near the antenna even if there is no ECR region in the discharge chamber. From Fig. 9, the electric field, which is high near the antenna, allows the electron energy $E_{\mathrm{e}}$ to increase to be about $10 \mathrm{eV}$. This is why ionization collisions occur in Case 2. A microwave discharge plasma source without magnetic field (without ECR) is suggested in Ref. 14).

The increase in $E_{\mathrm{e}}$ by the electric field near the antenna is low compared to that by the ECR. This happens because continuous acceleration caused by frequency synchronization between the electron cyclotron motion and microwaves is directly responsible for the increase in $E_{\mathrm{e}}$ by the ECR. On the other hand, the increase in $E_{\mathrm{e}}$ by the electric field near the antenna requires that the electric field has direction to accelerate the electrons, and has a high value. Near the antenna, the latter condition is easily satisfied, but the former condition is difficult to satisfy.

Figure 4 shows that the electric field strength $|E|$ in the ECR region is $9000 \mathrm{~V} / \mathrm{m}$ for Case 1 and $3000 \mathrm{~V} / \mathrm{m}$ for Case 
3. The difference between these cases causes different increases of $E_{\mathrm{e}}$ and ionization collisions. In antenna engineering, microwaves are radiated effectively when the antenna length is $\lambda / 4$, where $\lambda$ is the wavelength. For a microwave frequency of $4.25 \mathrm{GHz}, \lambda$ is calculated as $70 \mathrm{~mm}$. The antenna length from the exit of the back yoke to the antenna tip is approximately $\lambda / 8(8 \mathrm{~mm})$. Therefore, the performance of this antenna is inferior to a $\lambda / 4$ antenna in terms of providing the microwave energy to a distant region. However, even when the antenna do not have the appropriate length and configuration, generation of ionization collisions can be seen by the mechanism mentioned for Case 2 .

In summary, the most important factor in a microwave neutralizer is to shorten the distance between the ECR region and antenna. Inserting the antenna directly in the ECR region can produce plasma even when its length is $\lambda / 8$ and it hardly radiates microwaves. For practical application, a neutralizer must be small and light. Using an antenna of length $\lambda / 8$ satisfies these requirements, and results in improved performance of the microwave ion engine system.

\section{Conclusions}

The effects of magnetic field configuration and antenna position on plasma production were analyzed by the code coupling PIC method with FDTD method for a microwave neutralizer. The following conclusions were reached.

1. With respect to plasma production in the microwave neutralizer, electrons between the yokes are energized from the ECR and the high strength electric field near the antenna. The plasma is maintained by ionization collisions of energized electrons.

2. In the L-shaped antenna of the HAYABUSA neutralizer, the length is approximately $\lambda / 8$, so the microwave radiation is expected to be low. When the antenna length is $\lambda / 8$ and the ECR region is far from the antenna, the microwave do not sufficiently energize electrons. Therefore, the antenna must be close to the ECR region for effective plasma production.

\section{References}

1) Toki, K., Kuninaka, H., Nishiyama, K., Shimizu, Y. and Funaki, I.: Technological Readiness of Microwave Ion Engine System for MUSES-C Mission, 27th Int. Electric Propulsion Conf., IEPC-01174, 2001.

2) Funaki, I. and Kuninaka, H.: Overdense Plasma Production in a Low-power Microwave Discharge Electron Source, Jpn. J. Appl. Phys., 40 (2001), pp. 2495-2500.

3) Hirakawa, M. and Nakakita, M.: Simulation of Electron Cyclotron Resonance in a Microwave Discharge Ion Thruster, Trans. Jpn. Soc. Aeronaut. Space Sci., 47 (1999), pp. 267-271 (in Japanese).

4) Masui, H., Tanoue, T., Nakashima, H. and Funaki, I.: Development of an Electromagnetic Particle Code for Space Propulsion Application Using ECR Discharge, 23rd International Symposium on Space Technology and Science, Paper ISTS 2004-b-11, 2004.

5) Miyamoto, T., Mii, K., Nishijima, T., Ijiri, H., Takao, Y. and Nakashima, H.: Development of a New Microwave Discharge Type Ion Engine, Vacuum, 73 (2004), pp. 391-396.

6) Takao, Y., Masui, H., Miyamoto, T., Kataharada, H., Ijiri, H. and Nakashima, H.: Development of Small-Scale Microwave Discharge Ion Thruster, Vacuum, 73 (2004), pp. 449-454.

7) Opal, C. B., Peterson, W. K. and Beaty, E. C.: Measurements of Secondary-Electron Spectra Produced by Electron Impact Ionization of a Number of Simple Gases, J. Chem. Phys., 55 (1971), pp. 41004106.

8) Szabo, J. J., Jr., Martinez-Sanchez, M. and Batishchev, O.: Numerical Modeling of the Near-Anode Region in a TAL Thruster, AIAA 003653, 2000.

9) Yee, K. S.: Numerical Solution of Initial Boundary Value Problem Involving Maxwell's Equations in Isotopic Media, IEEE Trans. Antennas Propagat., 14 (1966), pp. 302-307.

10) Gropinath, V. P. and Grotjohn, T. A.: Three-dimensional Electromagnetic PIC Model of a Compact ECR Plasma Source, IEEE Trans. Plasma Sci., 23 (1995), pp. 602-608.

11) Adam, J. C., Heron, A. and Label, G.: Study of Stationary Plasma Thrusters Using Two-dimensional Fully Kinetic Simulations, Phys. Plasmas, 11 (2004), pp. 295-305.

12) Onodera, N., Takegahara, H., Nishiyama, K., Funaki, I. and Kuninaka, H.: Electron Emission Mechanism of Microwave Discharge Neutralizer, Trans. Jpn. Aeronaut. Space Sci., 49 (2001), pp. 27-31 (in Japanese).

13) Mur, G.: Absorbing Boundary Conditions for the Finite-Difference Approximation of the Time Domain Electromagnetic Field Equations, IEEE Trans. Electromag. Compat., 23 (1981), pp. 377-382.

14) Fusellier, C., Wartski, L., Aubert, J., Schwebel, C., Coste, Ph. and Chabrier, A.: A Microwave Plasma Cathode Electron Gun for Ion Beam Neutralization, Rev. Sci. Instrum., 69 (1998), pp. 1153-1155. 